\title{
Minor changes in fibre intake in the UK population between 2008/2009 and 2016/2017
}

\author{
Mathilde Gressier $\mathbb{1}^{1,2} \cdot$ Gary Frost $\mathbb{D}^{1}$
}

Received: 18 November 2020 / Revised: 15 April 2021 / Accepted: 21 April 2021 / Published online: 13 May 2021

(c) The Author(s) 2021. This article is published with open access

\begin{abstract}
The benefits of increasing populations' and individuals' fibre intake on non-communicable disease risk have been known and promoted for decades in the UK and in the world. Public health campaigns, including dietary recommendations, called populations to increase their consumption of whole grains, fruits and vegetables, while manufacturers increased the fibre content of their products. In particular, the SACN report in 2015 highlighted the importance of fibres for the UK population. We analysed trends in fibre consumption for the whole population, by age group and gender using the UK National Diet and Nutrition Survey from 2008/09 to 2016/17. We investigated changes in total fibre intake and calculated the contribution to fibre intake and time trends from each food group. We compared the fibre content of food groups between 2008/09 and 2016/17. We found that fibre intake remained fairly stable. While the fibre content of some cereal-based products increased, it decreased for potato-based products. All age groups derived increasing fibre from pasta and other cereal-based products, and decreasing fibre from potato products. Adults, but not children or adolescents derived more fibre from vegetables. This resulted in an increase in fibre intake in adults, but not in children or adolescents.
\end{abstract}

\section{Introduction}

Dietary fibres are component of plant-based foods that are not digested or absorbed in the small intestine [1] but fermented by micro-organisms in the gut. Their consumption is linked to various health benefits, including reducing the risk of cardiovascular diseases or type 2 diabetes and bowel cancer [2]. Intake of dietary fibres is consistently lower than recommended targets in all regions of the world. Intake is lowest in Asia ( 8-10 g/day) and highest in Oceania and western and eastern sub-Saharan Africa ( 16-20 g/day) [3]. The inadequate consumption of fibres was estimated to be responsible for about 100,000 deaths in 2017 globally [3]. In the UK, the importance of fibres in the diet was emphasized in the 2015 report of the Scientific Advisory

Mathilde Gressier

m.gressier18@imperial.ac.uk

1 Section for Nutrition Research, Department of Metabolism, Digestion and Reproduction, Faculty of Medicine, Imperial College London, London, UK

2 Centre for Health Economics \& Policy Innovation, Department of Economics \& Public Policy, Imperial College Business School, Imperial College London, London, UK
Committee on Nutrition (SACN, [1]). The report recommends a daily fibre intake of $30 \mathrm{~g} / \mathrm{day}$ or more for adults, a level from which the greatest health benefits were observed [1]. This message was promoted by various organizations promoting healthy diets, which designed new messages and campaigns to raise population awareness about the benefit of an adequate fibre intake. Most of these messages, such as the messages from the NHS, the British Heart Foundation or the British Nutrition Foundation, put emphasis on swapping refined grains to whole grains, as fibre and other healthful minerals are lost during the refinement of grains. The message to eat five fruits and vegetables a day was also reinforced [4-6]. In addition, food manufacturers formulate foods with increasing levels of fibres to improve their nutrient profile [7]. One strategy is to substitute refined grains with whole grains or to launch new products with whole grains. The focus on whole grains started in the 2000 s, with the introduction of a whole grain guideline in the US dietary guidelines [8], or the launch of the Danish Whole Grain Partnership in 2007 [9]. This increased focus on whole grains incentivised manufacturers to reformulate and formulate new products with whole grains [8]. There is currently limited evidence on the effect of this increased focus on dietary fibres in public health messages, policies, and food product innovations on fibre intake in the UK. The 
National Diet and Nutrition Survey (NDNS) rolling programme between 2008/09 and 2016/17 showed an increased intake of total fibres (of $0.3 \mathrm{~g} /$ year) [10], however, this figure does not capture reformulation, as the total fibre content of NDNS foods were added retrospectively in 2015/ 2016 [10]. In the US, fibre intake increased in children, adolescents, and adults aged over 51 years between 2001 and 2010 [11]. In this study, we investigated the evolution of fibre intake in the UK population between 2008/09 and 2016/17, with the SCAN recommendation published in 2015. Also, we investigated reformulation of food categories and possible changes in the food sources of fibres.

\section{Materials and methods}

Daily food intakes of the UK population were obtained from the NDNS from 2008/09 to 2016/17 [12]. We derived the nutrient composition of each food code (i.e. food reported in the survey) for each survey year from the Nutrient Databank, a regularly updated food composition table created from the McCance \& Widdowson table, made to match each survey year of the NDNS [12]. Food products were grouped into 17 food groups (listed in Table 1) defined in the McCance \& Widdowson table, separating potatobased products from vegetables to analyse these two food groups separately. The mean non-starch polysaccharide (NSP) fibre content of each food group (and of all foods) was calculated by averaging the fibre content of all foods appearing in each food group each year of the survey. Mean NSP fibre contents in years 2008/09 and 2016/17 were compared with the non-parametric Mann-Whitney $U$-test. To test changes in fibre content of paired food products (i.e. foods recorded in both surveys from 2008/09 and 2016/17), Wilcoxon signed-rank tests were used. Both tests were done first across all foods, then for the foods belonging to each food group.

We estimated the daily mean NSP fibre intake of the UK population in each of the 9 years of the survey. Trends in intake were estimated using linear regression, adjusted for demographic characteristics (age group, gender, BMI, ethnicity and equivalised income) and total energy intake. We added interaction terms between survey year and age group or sex to test the differential change by age or sex. We identified the food sources of NSP fibres for the UK population by averaging the contribution of each food group to participants' daily fibre intake (including consumers and non-consumers for each food group). Trends in the contribution of each food group to total fibre intake were estimated using linear regression, adjusted for the demographic and energy intake variables defined above. To take into account the sampling procedure of the NDNS and get estimates representative of the UK population, sampling weights were used in each procedure.

\section{Results}

Overall, there was no change in the mean NSP fibre content of all foods between 2008/09 and 2016/17 (mean content of $1.5 \mathrm{~g} / 100 \mathrm{~g}$ in the two years, non-significant Mann-Whitney $U$ test, Table 1). Nonetheless, there was a change in the composition of paired products (present in both 2008/09 and 2016/17, with a $p$-value $=0.0045$ (Wilcoxon test) (Table 1). The mean fibre content of some cereal-based products increased (such as bread: mean content of $3.6 \pm$ $1.8 \mathrm{~g} / 100 \mathrm{~g}$ in 2008/09, $3.8 \pm 1.9 \mathrm{~g} / 100 \mathrm{~g}$ in 2016/17), while one of the potato products decreased (mean content of $1.6 \pm 0.7 \mathrm{~g} / 100 \mathrm{~g}$ in $2008 / 09,1.3 \pm 0.7 \mathrm{~g} / 100 \mathrm{~g}$ in $2016 / 17$ ) (Table 1).

For the whole population, there was a marginal but significant increase in fibre intake (adjusted trend: $+0.06 \mathrm{~g} / \mathrm{year}$, $p$-value $=0.032$, adjusted for age group, sex, energy intake, BMI, ethnicity and equivalised income). However, after adding an interaction between age group and survey year, this significant increase was only found in adults (Fig. 1). Adding an interaction between sex and survey year revealed a significant increase in fibre intake only in males (adjusted trend: $+0.09 \mathrm{~g} /$ day/year, $p=0.041$ ), but not in females. Similar results were obtained when the regressed term was fibre intake for $10 \mathrm{MJ}$ (the adjusted trend for males: $+0.10 \mathrm{~g} / 10 \mathrm{MJ} /$ year, $p=0.026)$.

Cereal-based products (bread, pasta, rice and other cereals, breakfast cereals, biscuits, pastries and puddings) were the first contributor to NSP fibres intake in children, adolescents and adults. Bread alone corresponded to $\sim 20 \%$ of fibre intake in children, adolescents and adults (Fig. 2). The vegetable was the second contributor, followed by potato products and fruits. For all age groups, the contribution of potato products to NSP fibres decreased between 2006/07 and 2016/17, while the contribution from pasta, rice and other cereals increased. In adults, the contribution of vegetables increased (Fig. 2C), but not for children (Fig. 2A) or adolescents (Fig. 2B).

\section{Discussion}

Although multiple initiatives were implemented to increase fibre intake in the UK population [1], intake remained fairly stable. A small increase of $0.09 \mathrm{~g} /$ day per survey year was found in adults, small in the comparison to the gap between estimated mean fibre intake in adults $(14.9 \mathrm{~g} / \mathrm{day}, \mathrm{SE}=0.5)$ and recommendations (30 g/day). Several reasons can explain this resistance to add fibre-rich food to diets. First, 


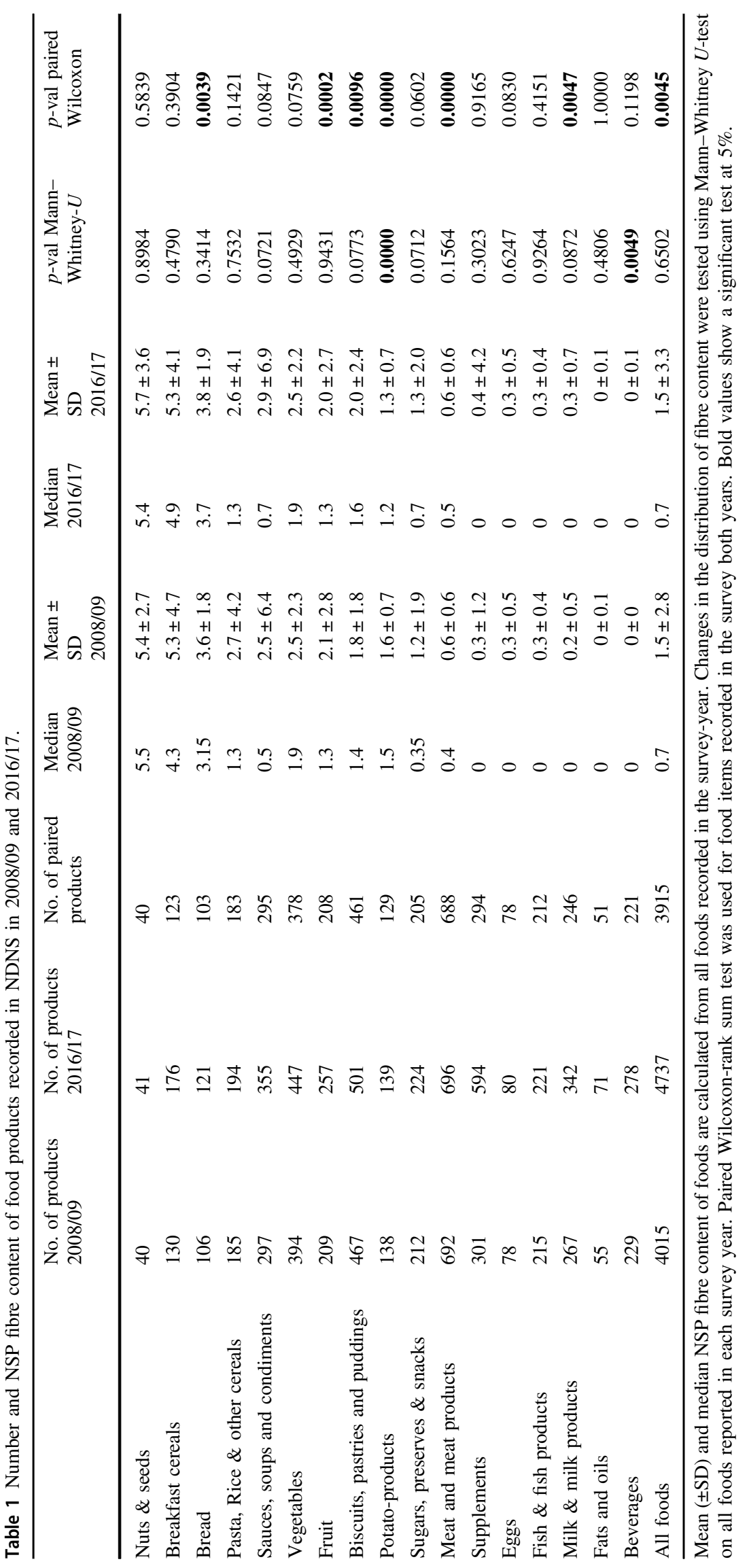


Fig. 1 Trend in mean fibre intake of the UK population between 2008/09 and 2016/17, by age group. The graph shows mean fibre intake and 95\% confidence intervals for each age group and each survey year of the NDNS. Trendlines were plotted for each age group. Corresponding estimates for the time trends are shown on the right of the graph (corresponding to the interaction term between survey year and age group). Asterisks (*) indicates a significant estimate at a $5 \%$ level.

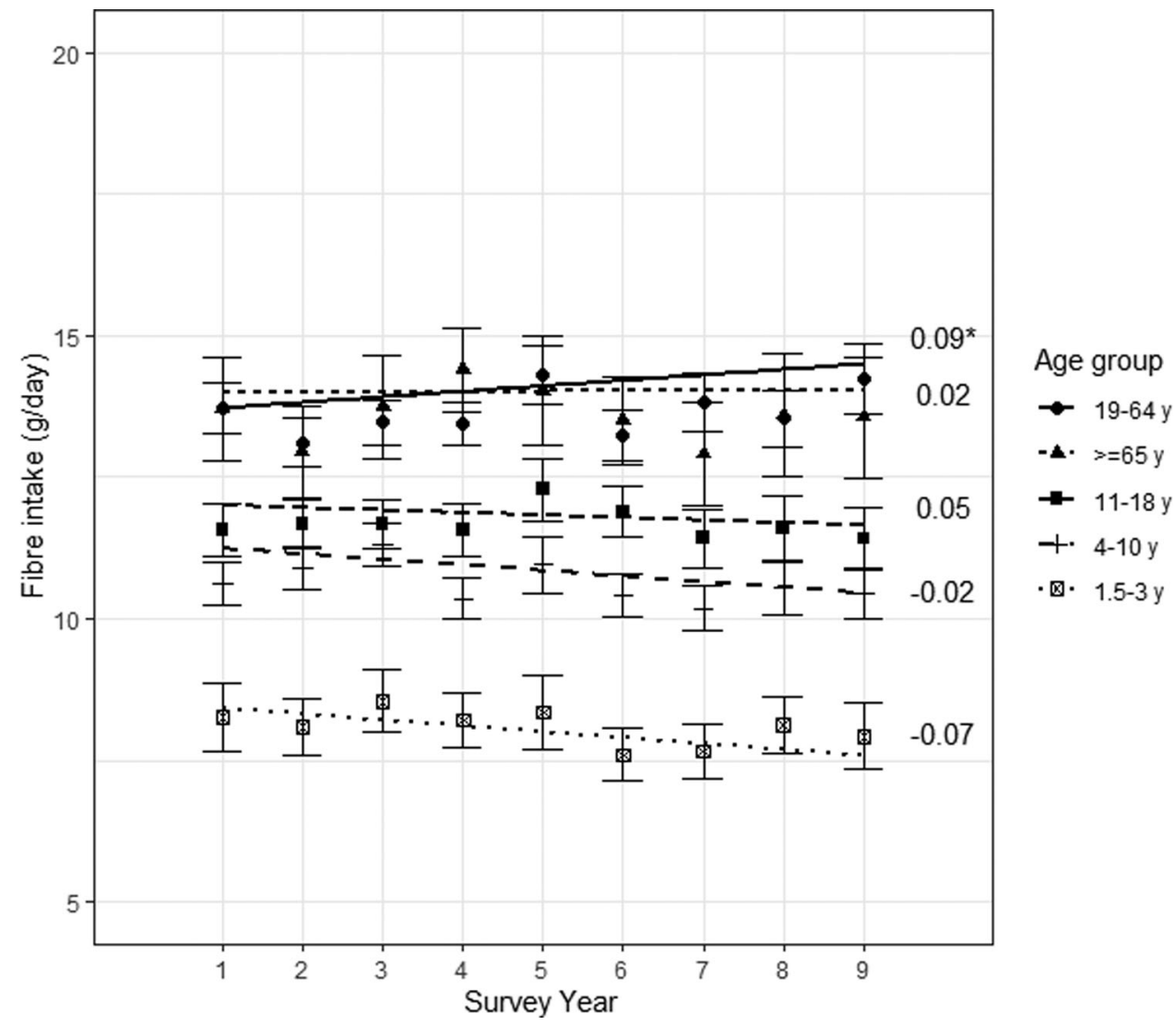

the sensory profile of whole-grain products is not accepted by some consumers, unlikely to switch from refined grainto whole grain products [13]. Also, some consumers link starchy foods to weight gain or digestion discomfort, hence do not want to increase their intake of fibre-containing starchy foods [14].

For all age groups, there was a shift from fibres from potato products to pasta, rice, and other cereals. Beyond fibre intake, this increased consumption of cereal-based products can have a varying impact on other nutrients if the shift was in favour of whole or refined grain products. Refined grain products have fewer vitamins and minerals than whole-grain products; despite refined flour being enriched in vitamins and minerals, wholemeal bread remains a better source of vitamins and minerals than white bread [15].

The evaluation of trends in intake from NDNS by Public Health England (PHE) found increased intake of total fibres (without considering the effect of reformulation) (of $0.3 \mathrm{~g} /$ year) between 2006/07 and 2016/17 [10], while we found an increase in NSP fibres (including the effect of reformulation) of $0.06 \mathrm{~g} / \mathrm{year}$. The difference between these results can come from the increased consumption of foods containing non-NSP fibres such as resistant starch, inulin or polydextrose. These fibres are increasingly used as a food supplement or added to food products to improve their sensory or nutritional characteristics [7]. Thus, our estimates of reformulation based on NSP fibres are likely to underestimate the change in total fibres. The NDNS report also found a significant increase in total fibre intake for adult men but not for women.

In conclusion, this study shows that fibre intake remained fairly constant in the 2010s, despite information campaigns about how to increase one's fibre intake, and actions from manufacturers to increase the fibre content of cereal-based products. The source of fibre changed, with adults deriving more fibre from vegetables and less from potato-based products. This could be the effect of public health campaigns highlighting the importance of an adequate vegetable intake. However, this favourable increase in fibre from vegetables was not observed in children and adolescents. Children and adolescents derived more fibre from pasta and other cereal-based products, but this was not enough to be translated into a change in fibre intake. Cereal-based products have a higher fibre density than vegetables, but vegetables are nutrient-dense and have a low energy density, making them essential in children diets. Although the reformulation of cereal-based products to increase their fibre content and their promotion is laudable to improve fibre intake of the population, efforts should also focus on 

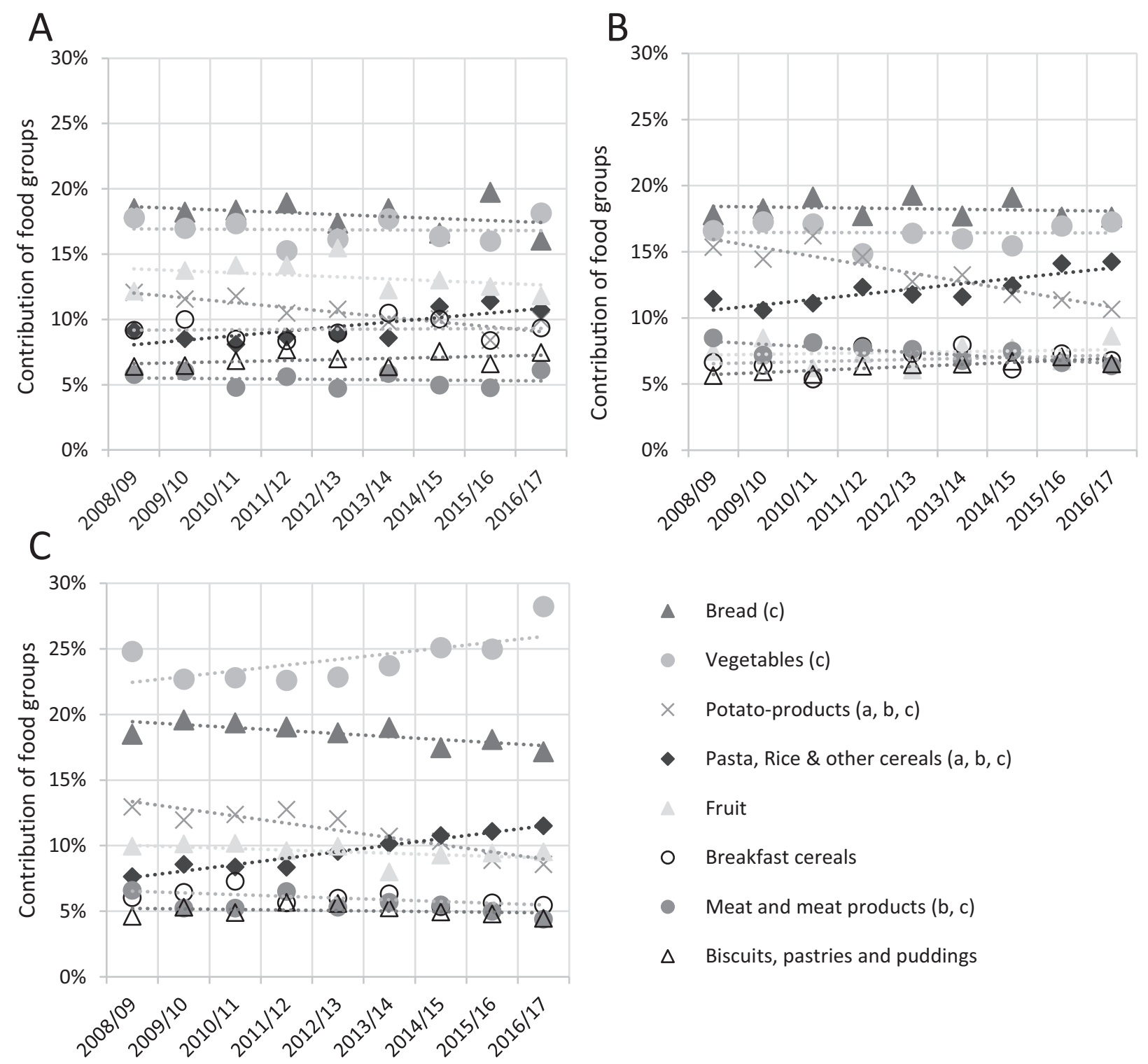

\author{
$\triangle \operatorname{Bread}(\mathrm{c})$ \\ Vegetables (c) \\ $\times$ Potato-products $(a, b, c)$ \\ - Pasta, Rice \& other cereals $(a, b, c)$ \\ Fruit \\ O Breakfast cereals \\ - Meat and meat products (b, c) \\ $\Delta \quad$ Biscuits, pastries and puddings
}

Fig. 2 Mean contribution of each food group to non-starch polysaccharide (NSP) intake between 2008/09 (Year 1) and 2016/17 (Year 9) by age group. Contributions for children aged 4--10 years and shown in (A), for adolescents aged 11-18 years in (B) and adults

increasing vegetable consumption in children and adolescents.

\section{Compliance with ethical standards}

Conflict of interest $\mathrm{MG}$ is the recipient of an unconditional $\mathrm{PhD}$ Studentship grant from Societé des Produits Nestlé, supervised by GF. Societé des Produits Nestlé has no role in the study design, data collection and analysis, or preparation of the protocol and research paper. The Nutrition Research Group is funded by grants from the MRC, BBSRC, NIHR, an Integrative Mammalian Biology (IMB) Capacity Building Award, and is supported by the NIHR Biomedical Research Centre Funding Scheme. GF is an NIHR senior investigator. The views expressed are those of the author(s) and not necessarily those of the NHS, the NIHR or the Department of Health. aged 19-64 years old (C). Letters 'a', 'b', or 'c' in the legend indicates a significant time trend (significant effect of year on mean fibre intake), for the Contributions for children aged 4--10 years and shown in (A), for adolescents aged 11-18 years in (B) and adults aged 19-64 years old (C).

Publisher's note Springer Nature remains neutral with regard to jurisdictional claims in published maps and institutional affiliations.

Open Access This article is licensed under a Creative Commons Attribution 4.0 International License, which permits use, sharing, adaptation, distribution and reproduction in any medium or format, as long as you give appropriate credit to the original author(s) and the source, provide a link to the Creative Commons license, and indicate if changes were made. The images or other third party material in this article are included in the article's Creative Commons license, unless indicated otherwise in a credit line to the material. If material is not included in the article's Creative Commons license and your intended use is not permitted by statutory regulation or exceeds the permitted use, you will need to obtain permission directly from the copyright holder. To view a copy of this license, visit http://creativecommons. org/licenses/by/4.0/. 


\section{References}

1. Scientific Advisory Committee on Nutrition. Carbohydrates and health. London: Scientific Advisory Committee on Nutrition; 2015.

2. Stephen AM, Champ MM-J, Cloran SJ, Fleith M, van Lieshout L, Mejborn $\mathrm{H}$, et al. Dietary fibre in Europe: current state of knowledge on definitions, sources, recommendations, intakes and relationships to health. Nutr Res Rev. 2017;30:149-90.

3. Afshin A, Sur PJ, Fay KA, Cornaby L, Ferrara G, Salama JS, et al. Health effects of dietary risks in 195 countries, 1990-2017: a systematic analysis for the Global Burden of Disease Study 2017. Lancet. 2019;393:1958-72.

4. NHS UK. How to get more fibre into your diet. 2019. https://www.nhs.uk/live-well/eat-well/how-to-get-more-fibre-intoyour-diet/. Accessed 31 Aug 2020.

5. British Heart Foundation. 7 easy ways to add more fibre to your diet-heart matters-BHF. Hear Matters. 2019. https://www.bhf. org.uk/informationsupport/heart-matters-magazine/nutrition/fibre/ fibre-swaps. Accessed 31 Aug 2020.

6. British Nutrition Foundation. Dietary fibre. 2020. https://www.nutrition. org.uk/healthyliving/basics/fibre.html. Accessed 31 Aug 2020.

7. Foschia M, Peressini D, Sensidoni A, Brennan CS. The effects of dietary fibre addition on the quality of common cereal products. J Cereal Sci 2013;58:216-27.
8. Mancino L, Kuchler F, Leibtag E. Getting consumers to eat more whole-grains: the role of policy, information, and food manufacturers. Food Policy. 2008;33:489-96.

9. Lourenço S, Hansen GL, Stærk B, Frank P, Petersen CT. The whole grain partnership-how a public-private partnership helped increase whole grain intake in Denmark. Cereal Foods World. 2019;64:1-9.

10. Public Health England, Food Standards Agency. Years 1 to 9 of the rolling programme (2008/2009-2016/2017): time trend and income analyses. 2019.

11. McGill CR, Fulgoni VL, Devareddy L. Ten-year trends in fiber and whole grain intakes and food sources for the united states population: National health and nutrition examination survey 2001-2010. Nutrients. 2015;7:1119-30.

12. NatCen Social Research, MRC Elsie Widdowson Laboratory. National diet and nutrition survey years 1-9, 2008/09-2016/17. 2019. https://doi.org/10.5255/UKDA-SN-6533-15.

13. Li M, Ho KKHY, Hayes M, Ferruzzi MG. The roles of food processing in translation of dietary guidance for whole grains, fruits, and vegetables. Annu Rev Food Sci Technol. 2019;10:569-96.

14. Hooper B, Spiro A, Stanner S. $30 \mathrm{~g}$ of fibre a day: an achievable recommendation? Nutr Bull. 2015;40:118-129.

15. Lockyer S, Spiro A. The role of bread in the UK diet: an update. Nutr Bull 2020;45:133-164. 